\title{
Insulin regulation of glucose turnover and lipid levels in obese children with fasting normoinsulinaemia
}

\author{
L. D. Monti ${ }^{1}$, P. Brambilla ${ }^{2}$, I. Stefani ${ }^{1}$, A. Caumo ${ }^{1}$, F. Magni $^{3}$, R. Poma ${ }^{1}$, L. Tomasini ${ }^{2}$, G. Agostini ${ }^{2}$, M. Galli-Kienle ${ }^{4}$, \\ C. Cobelli ${ }^{5}$, G. Chiumello ${ }^{2}$, G. Pozza ${ }^{1}$
}

${ }^{1}$ Istituto Scientifico H. San Raffaele, Cattedra di Clinica Medica, Universita' di Milano, Milano, Italy

${ }^{2}$ Istituto Scientifico H. San Raffaele, Cattedra di Clinica Pediatrica, Universita' di Milano, Milano, Italy

${ }^{3}$ Laboratorio di Spettrometria di Massa, Universita' di Milano, Milano, Italy

${ }^{4}$ Dipartimento di Chimica e Biochimica Medica; Universita' di Milano, Milano, Italy

${ }^{5}$ Dipartimento di Elettronica ed Informatica, Universita' di Padova, Padova, Italy

\begin{abstract}
Summary To evaluate the early metabolic alterations induced by obesity, we studied glucose turnover and lipid levels in obese children with fasting normoinsulinaemia. Two experimental protocols were carried out. Protocol I consisted of a euglycaemic glucose clamp at two rates of insulin infusion. Protocol II was similar to protocol I except for a variable lipid infusion used to maintain basal non-esterified fatty acid (NEFA) levels. During protocol I, the glucose disappearance rates were lower in obese children, while no differences were found in hepatic glucose release. NEFA response to insulin was not substantially altered in obese children either at low or high insulin infusion. During protocol II, the NEFA clamp induced a $25 \%$ reduction in peripheral insulin sensitivity in control children whereas no changes were observed in obese children. Interestingly, lipid infusion in control children was not sufficient to reproduce
\end{abstract}

the same degree of insulin resistance observed in obese children, suggesting that NEFA are only one of the determinants of insulin resistance at this stage of obesity. In conclusion, the present study provides a portrait of glucose metabolism and lipid levels in normoinsulinaemic obese children. Our results document that peripheral insulin resistance is the first alteration at this stage of obesity, whereas an increase in insulin secretion and a defect in the inhibition of hepatic glucose release by insulin may develop at a later stage. In addition, primarily receptor and post-receptor defects and some alterations of NEFA metabolism are likely to coexist in the induction of insulin resistance at this stage of obesity. [Diabetologia (1995) 38: 739-747]

Key words Obesity, children, insulin action, lipids, glucose turnover.
Obesity in adults has long been associated with multiple disorders of glucose-insulin and lipid homeostasis [1-6]. In particular, a marked reduction of glucose disposal has been demonstrated in obese subjects reflecting primarily muscle insensitivity to insulin action [7-9]. In addition, it has been shown that the im-

Received: 30 May 1994 and in final revised form: 4 January 1995

Corresponding author: Dr. L.D. Monti, Department of Medicine, Istituto Scientifico H. San Raffaele, Via Olgettina 60, I20132 Milan, Italy

Abbreviations: NEFA, Non-esterified fatty acids; FFM, fat free mass; APE, atom percent excess; HGR, hepatic glucose release; $R_{a}$, glucose appearance; $R_{d}$, glucose disappearance; $C V$, coefficient of variation. pairment in insulin action in obese subjects is correlated to an increased non-esterified fatty acid (NEFA) oxidation (glucose-fatty acid cycle) [10-20]. However, since all the previous studies have been performed in patients with marked metabolic alterations related to the long duration of obesity, it remains to be elucidated whether in the early phase of obesity hyperinsulinaemia or a defect in glucose disposal is the first event, and whether NEFA levels play a crucial role in this respect.

Children, at the early onset of obesity and in a phase of rapid weight gain, appear to be an interesting group for studying early alterations in human obesity. However, very few studies have been performed in this group. Bougneres et al. [21] studied seven non-diabetic grossly obese children and found a lower glucose disposal and a higher endogenous glucose produc- 
Table 1. Clinical characteristics and anthropometric data of obese and control children

\begin{tabular}{lrc}
\hline & Obese $(n=14)$ & Control $(n=13)$ \\
\hline Age (years) & $11.6 \pm 0.6$ & $12.4 \pm 0.7$ \\
Body weight $(\mathrm{kg})$ & $66.4 \pm 4.1$ & $46.2 \pm 3.8$ \\
Ideal body weight $(\%)$ & $159.1 \pm 3.5$ & $110.3 \pm 1.8$ \\
Body mass index $\left(\mathrm{kg} / \mathrm{m}^{2}\right)$ & $28.5 \pm 0.6$ & $19.9 \pm 0.8$ \\
Fat free mass $(\mathrm{kg})$ & $42.5 \pm 3.5$ & $33.7 \pm 2.2$ \\
Fat free mass (\% of BW) & $59.4 \pm 2.3$ & $76.0 \pm 2.1$ \\
Fat mass (kg) & $23.9 \pm 3.7$ & $12.5 \pm 2.7$ \\
Fat mass (\% of BW) & $40.7 \pm 2.5$ & $24.0 \pm 2.1$ \\
Duration of obesity (years) & $5.5 \pm 0.6$ & - \\
\hline
\end{tabular}

Mean \pm SEM

tion. Of note is that fasting hyperinsulinaemia was present suggesting that a relatively long period of insulin resistance was already established in the obese children. More recently, Le Stunff and Bougneres [22] have studied obese normoinsulinaemic children and demonstrated an increased lipid oxidation conditioning a decreased glucose oxidation in the fasting period.

In the present study our interests were focused on the evaluation of glucose turnover and lipid levels in a group of obese children with a recent onset of obesity and a rapid weight gain but in the presence of normal insulin levels in the fasting state. The group was compared to age-matched normal weight control children in two protocols. The first protocol (protocol I) consisted of a study on glucose turnover and lipid levels by the euglycaemic hyperinsulinaemic clamp technique at two insulin infusion rates. The second protocol (protocol II) was similar to the first one except for a variable lipid infusion aimed at maintaining NEFA at the basal levels. The NEFA clamp levels permitted assessment of the effect of NEFA levels on glucose turnover at this early stage of obesity.

\section{Subjects and methods}

Subjects. Fourteen children with simple obesity ( 8 males, $6 \mathrm{fe}-$ males) and 13 control children ( 6 males, 7 females) were studied. Simple obesity was defined as the absence of genetic or endocrinologic abnormalities in the presence of normal stature. Parents freely accepted having their children participate in the study after a full explanation of the aims and the potential side effects or risks. The study was also approved by the Institutional Ethical Review Board.

Anthropometric data and fasting levels. Clinical characteristics and body composition of obese and control children are depicted in Table 1.

Obese and control children were prepubertal as it is well known that puberty decreases insulin sensitivity $[23,24]$. Boys had testes volume less than $4 \mathrm{ml}$, and pubic hair stage P1. No breast or pubic hair development was seen in girls.

Since we were interested in studying the early stage of obesity, all the obese children chosen had exhibited signs of the disease for no longer than 6 years with a rapid weight gain in the last year $(9.0 \pm 2.0 \mathrm{~kg})$. The onset of obesity was dated from when body weight exceeded $120 \%$ ideal body weight using growth curve charts recorded from the children's births. Mean ponderal excess was $59.1 \pm 3.5 \%$ with respect to ideal body weight, according to Tanner and Whitehouse [25]. The evaluation of fat mass was performed with multiple skin-fold measurements (triceps, biceps, subscapular, suprailiac) with a Harpenden caliper [26] by the same trained investigator to reduce the relative inaccuracy of the method $[27,28]$. Fat free mass (FFM) was then calculated by subtracting adipose tissue mass from total body weight. The obese children were not dieting before the start of the study and during the period of study all subjects consumed a normo-caloric diet for age and sex according to Italian Dietary Recommendations [29] consisting of $1000 \mathrm{kcal}$ plus $100 \mathrm{kcal}$ per year of age, with $55 \%$ carbohydrate. They all had fasting normoglycaemia and normoinsulinaemia as compared to levels found in control children and to previously reported data $[30,31]$ and none had a family history of diabetes. Glucose and insulin responses at $120 \mathrm{~min}$ after oral glucose tolerance test (OGTT) were higher in obese than control children (Table 2) but all the obese children had normal glucose tolerance according to the criteria of the Diabetes Data Group [32].

Experimental protocol. The children were admitted to the hospital at 08.00 hours, after an overnight fast. After $30 \mathrm{~min}$ of bed rest and familiarization with the testing procedures and the equipment used, a 20 gauge Abbocath (Venisystems, Abbot Ireland Ltd., Sligo, Republic of Ireland) was inserted in an antecubital vein for intravenous infusions. A similar cannula was retrogradely inserted into a vein of the dorsum of the hand and the hand placed into a plexiglass box heated at $55^{\circ} \mathrm{C}$ for arterialising blood. The sampling cannula was kept patent by a slow $0.9 \% \mathrm{NaCl}$ infusion.

Studies consisted of euglycaemic glucose clamps at either a low insulin infusion rate $\left(0.4 \mathrm{mU} \cdot \mathrm{kg}^{-1} \cdot \mathrm{min}^{-1}\right)$ or at a high insulin infusion rate $\left(1.7 \mathrm{mU} \cdot \mathrm{kg}^{-1} \cdot \mathrm{min}^{-1}\right)$ and each obese or control subject was randomly allocated to only one insulin infusion rate for ethical reasons. The studies were performed as paired tests: e.g. one experiment with insulin infusion (protocol I) and a second experiment with insulin plus lipid infusion (protocol II). The order of the two experiments was randomised, and the two tests were performed one week apart. Except for the lipid infusion, all test procedures were identical for protocol I and II.

Each study began with a primed $(5 \mathrm{mg} / \mathrm{kg})$, continuous $\left(0.05 \mathrm{mg} \cdot \mathrm{kg}^{-1} \cdot \mathrm{min}^{-1}\right)$ infusion of $\left[6,6-^{2} \mathbf{H}_{2}\right]$-glucose that continued until the end of the study. After a 2 -h tracer equilibration period, samples were drawn at $-20,-15,-10,-5,-1$ min for subsequent determination of basal glucose turnover. At 0 min, a 3-h euglycaemic, hyperinsulinaemic clamp at either low or high insulin infusion was begun. After initiation of the insulin infusion, glucose concentration was clamped at the basal level using a variable $20 \%$ dextrose infusion. $\left[6,6-{ }^{2} \mathrm{H}_{2}\right]$-glucose was added to the infused glucose in order to minimize the changes in atom percent excess (APE) during the experiment (hot-GINF technique) [33]. Enrichment of exogenous glucose infusate was calculated by providing estimates of hepatic glucose release (HGR) at basal steady state and of both hepatic glucose release and exogenous glucose infusion rate at the end of the clamp as suggested by Finegood et al. [33]. The enrichment of the infusate was kept constant throughout both insulin infusions and was a compromise between the calculated value for each clamp study. In protocol II, a variable infusion of Intralipid $20 \%$ (Kabivitrum AB, Stockolm, Sweden) was used to clamp NEFA concentration to the level measured in 
Table 2. Hormonal and metabolic fasting levels in obese and control children

\begin{tabular}{|c|c|c|c|}
\hline & $\begin{array}{l}\text { Obese } \\
(n=14)\end{array}$ & $\begin{array}{l}\text { Control } \\
(n=13)\end{array}$ & $p$-value \\
\hline \multirow{8}{*}{$\begin{array}{l}\text { Blood glucose } \\
\text { (mmol//) } \\
\text { Plasma insulin } \\
\text { (pmol/l) } \\
\text { Serum C-peptide } \\
\text { (nmol/l) } \\
\text { Plasma glucagon } \\
\text { (mg/l) } \\
\text { Plasma NEFA } \\
\text { (mmol/l) } \\
\text { Serum triglyceride } \\
(\mathrm{mg} / \mathrm{dl}) \\
\text { Blood } \beta \text {-OH-butyrate } \\
(\mu \mathrm{mol} / \mathrm{l}) \\
\text { Blood glycerol } \\
(\mu \mathrm{mol} / \mathrm{l})\end{array}$} & $4.8 \pm 0.1$ & $4.9 \pm 0.1$ & \\
\hline & $60.0 \pm 5.4$ & $52.8 \pm 4.2$ & \\
\hline & $0.52 \pm 0.03$ & $0.45 \pm 0.03$ & \\
\hline & $77.9 \pm 5.4$ & $86.2 \pm 7.0$ & \\
\hline & $0.72 \pm 0.04$ & $0.68 \pm 0.03$ & \\
\hline & $64.6 \pm 7.6$ & $50.6 \pm 5.0$ & \\
\hline & $449.6 \pm 74.0$ & $261.9 \pm 42.5$ & $<0.05$ \\
\hline & $61.1 \pm 7.4$ & $56.8 \pm 4.8$ & \\
\hline \multirow{3}{*}{$\begin{array}{l}\text { Oral glucose tolerance } \\
\text { 2-h Blood glucose } \\
(\mathrm{mmol} / \mathrm{l}) \\
\text { 2-h Plasma insulin } \\
\text { (pmol/l) }\end{array}$} & test (mean \pm & & \\
\hline & $6.4 \pm 1.1$ & $5.0 \pm 0.8$ & $<0.05$ \\
\hline & $335 \pm 141$ & $166 \pm 37$ & $<0.05$ \\
\hline
\end{tabular}

obese children during the fasting period. The lipid infusion was changed every $15-20 \mathrm{~min}$ on the basis of NEFA levels determined by Cobas Fara II (Roche, Basel, Switzerland).

Calculation of glucose iurnover. The rates of appearance $\left(R_{a}\right)$ and disappearance $\left(R_{d}\right)$ of glucose were calculated using Steele's model [34] by using the stable isotope equivalent of specific activity, i.e. the tracer-to-tracee ratio. Tracer-to-tracee ratio was calculated from APE using the kinetic formalism developed perviously [35]. A pool fraction of 0.65 and a total distribution volume of $260 \mathrm{ml} / \mathrm{kg}$ were used. HGR was calculated by subtracting from $R_{a}$ the exogenous dextrose infusion. Glucose and APE values were subjected to smoothing using the optimal segments technique [36].

Insulin dose-response curves for $R_{d}$ and $H G R$. Insulin dose-response characteristics for stimulation of $R_{d}$ and suppression of HGR were generated by plotting the rates of $R_{d}$ and hepatic release calculated in the last $30 \mathrm{~min}$ of each insulin infusion vs insulin concentration. Since $R_{d}$ reflects the effect of insulin on glucose-utilizing tissues, whereas HGR is mainly regulated by insulin and liver insulin sensitivity, we chose to express $R_{d}$ as the disappearance rate per unit of lean body mass per unit of time (mg $\cdot \mathrm{kgFFM}^{-1} \cdot \mathrm{min}^{-1}$ ) and HGR as the total amount of glucose produced per min $(\mathrm{mg} / \mathrm{min})$.

Calculation of insulin effect on NEFA levels. In order to characterize the dynamics of insulin action on NEFA levels during protocol I, each individual NEFA decay curve was modelled as a delayed exponential decay from an initial level, NEFA, to a plateau level, NEFA ${ }_{p}$, as previously described [37] taking into account the time constant of NEFA decay $(\mathrm{k})$, and the time delay (in minutes) between the start of insulin infusion and the beginning of NEFA decrease $(\Delta t)$. Parameters NE$\mathrm{FA}_{\mathrm{i}}, \mathrm{NEFA}_{\mathrm{p}}, \mathrm{k}$, and $\Delta \mathrm{t}$ were estimated by non-linear leastsquares technique [38]. The absolute decrements in NEFA levels due to insulin infusion were calculated as NEFA $_{i}-$ NEFA $_{p}$ while the percentage inhibition of NEFA levels due to insulin infusion was calculated as $100^{*}\left(\mathrm{NEFA}_{\mathrm{i}}-\mathrm{NEFA}_{\mathrm{p}}\right) / \mathrm{NEFA}_{\mathrm{i}}$. The responsiveness of NEFA to insulin was measured by the rate constant of the exponential decay, $\mathrm{k}$.

Analytic determinations. Plasma glucose was measured by a glucose oxidase method (Beckman Glucose Analyzer II, Beckman Instruments Inc., Palo Alto, Calif., USA). Isotopic enrichment of $\left[6,6-{ }^{2} \mathrm{H}_{2}\right]$-glucose was determined by GC-MS after deproteinization and glucose conversion into the aldonitrile pentaacetate derivative [39].

Serum insulin, C-peptide and glucagon were determined by specific radio-immunoassays. In particular, insulin with Insulin I125 Ria kit (Incstar Corporation, Stillwater, Minn., USA); Cpeptide with C-peptide Double Antibody kit (Diagnostic Products Corporation, Los Angeles, Calif., USA) and glucagon with Ria Mat Glucagon kit (Byk Gulden, Milan, Italy). In our laboratory the intra-assay coefficients of variation (CVs) for the above determinations were 3,3 and $5 \%$ while inter-assay CVs were 5,5 and $7 \%$.

NEFA levels were assayed using an enzymatic technique on Cobas Fara Centrifugal Analyzer (Cobas Fara II, Roche) [40].

Blood $\beta$-OH-butyrate and glycerol levels were measured on blood deproteinised with $5 \%$ percloric acid using enzymatic spectrofluorimetric techniques [41]. The intra-assay CVs for the above determinations were 2.9 and $5.2 \%$, respectively, while inter-assay CVs were 4.0 and $5.8 \%$, respectively.

\section{Statistical analysis}

All data are presented as mean \pm SEM. Comparisons between obese and control children were made by means of analysis of variance followed by Scheffe $F$-test when appropriate. Comparisons between protocol I vs protocol II were performed within each of the obese and control groups by using paired $t$ test.

\section{Results}

Fasting period. No significant differences were found between the fasting periods of protocols I and II in either group. Obese children were normoglycaemic and normoinsulinaemic as compared to control children. Plasma glucagon, plasma NEFA and serum triglyceride levels were not significantly different in the two groups (Table 2 and 3 ). Blood $\beta$-OH-butyrate levels were higher in obese than control children.

The basal $\mathrm{R}_{\mathrm{d}}$ was significantly lower in obese children when compared to control children $(3.2 \pm 0.2$ vs $\left.4.3 \pm 0.2 \mathrm{mg} \cdot \mathrm{kgFFM}^{-1} \cdot \mathrm{min}^{-1} ; p<0.05\right)$. By contrast, basal HGR was similar in obese and control children (136.2 \pm 5.4 vs $144.7 \pm 6.3 \mathrm{mg} / \mathrm{min}, \mathrm{NS})$.

Clamp period. No significant differences were demonstrated in the plasma glucose levels of obese or control children with either low or high insulin infusion rates during protocol I and II. The coefficients of variation of glucose concentration were always below $5 \%$. Peripheral insulin levels were slightly higher in obese children than control children both during the low and high insulin infusion rates but the differ- 
Table 3. Parameters of plasma NEFA decline during euglycaemic clamp

\begin{tabular}{|c|c|c|c|c|}
\hline & \multicolumn{2}{|l|}{ Low insulin } & \multicolumn{2}{|l|}{ High insulin } \\
\hline NEFA initial (mmol/l) & $0.85 \pm 0.05$ & $0.74 \pm 0.05$ & $0.65 \pm 0.07$ & $0.78 \pm 0.06$ \\
\hline NEFA decrement (mmol/l) & $0.53 \pm 0.08$ & $0.56 \pm 0.06$ & $0.52 \pm 0.09$ & $0.65 \pm 0.06$ \\
\hline NEFA inhibition (\%) & $60.4 \pm 5.3^{\mathrm{a}}$ & $75.8 \pm 2.2$ & $75.8 \pm 4.1$ & $82.9 \pm 1.8$ \\
\hline$\Delta \mathrm{t}(\mathrm{min})$ & $13.3 \pm 1.9$ & $11.2 \pm 1.5$ & $14.6 \pm 1.7$ & $10.9 \pm 1.3$ \\
\hline
\end{tabular}

Mean \pm SEM

${ }^{a} p<0.05$ between groups

Table 4. Steady-state hormonal levels at the end of the clamp period

\begin{tabular}{|c|c|c|c|c|c|c|c|c|}
\hline & \multicolumn{4}{|l|}{ Low insulin } & \multicolumn{4}{|l|}{ High insulin } \\
\hline & \multicolumn{2}{|c|}{ Obese $(n=7)$} & \multicolumn{2}{|c|}{ Control $(n=7)$} & \multicolumn{2}{|c|}{ Obese $(n=7)$} & \multicolumn{2}{|c|}{ Control $(n=6)$} \\
\hline & Protocol I & Protocol II & $\overline{\text { Protocol I }}$ & Protocol II & Protocol I & Protocol II & Protocol I & Protocol II \\
\hline $\begin{array}{l}\text { Serum insulin } \\
(\mathrm{pmol} / \mathrm{l})\end{array}$ & $176 \pm 12$ & $228 \pm 18$ & $160 \pm 14$ & $168 \pm 15$ & $687 \pm 29$ & $714 \pm 42$ & $594 \pm 32$ & $621 \pm 43$ \\
\hline $\begin{array}{l}\text { Serum C-peptide } \\
(\mathrm{nmol} / \mathrm{l})\end{array}$ & $0.17 \pm 0.07$ & $0.23 \pm 0.07$ & $0.20 \pm 0.03$ & $0.20 \pm 0.03$ & $0.23 \pm 0.03$ & $0.36 \pm 0.07$ & $0.23 \pm 0.03$ & $0.36 \pm 0.13$ \\
\hline $\begin{array}{l}\text { Plasma glucagon } \\
\text { (ng/l) }\end{array}$ & $70.6 \pm 9.0$ & $66.1 \pm 10.7$ & $74.0 \pm 12.4$ & $84.4 \pm 13.2$ & $69.1 \pm 10.7$ & $66.0 \pm 10.9$ & $69.6 \pm 6.5$ & $79.6 \pm 14.2$ \\
\hline
\end{tabular}

Mean \pm SEM

ences failed to reach a statistical significance. Lipid infusion did not cause significant changes in the final steady-state insulin, C-peptide and glucagon levels (Table 4). Percentages of inhibition of C-peptide secretion were significantly lower in control children after lipid infusion at high insulin infusion $(28.2 \pm 21.9$ vs $54.8 \pm 7.9 \%$ protocol II vs protocol I; $p<0.01)$.

Figure 1 shows plasma tracer enrichment (APE) at basal (from -20 to $0 \mathrm{~min}$ ) and final (from 150 to $180 \mathrm{~min}$ ) steady-state during protocols I and II. In the fasting period before the low and high insulin infusion clamp, APE levels were $2.65 \pm 0.13$ and $2.48 \pm 0.18$ in the obese children and $1.65 \pm 0.15$ and $1.62 \pm 0.11$ in the control children. These levels were maintained virtually constant during the clamp period in all the groups studied. Only in control children at low insulin infusion the APE levels measured at the end of the clamp period were slightly but not significantly higher than during the fasting period. During protocol II the APE levels were superimposable on those obtained during protocol I.

Glucose disappearance. The dose-response curves of $\mathrm{R}_{\mathrm{d}}$, as reported in Figure 2 (upper panels), suggest that a peripheral insulin resistance is present in obese children. During protocol $I, R_{d}$ values were reduced in obese children by $30 \%(4.5 \pm 0.3$ vs $\left.6.2 \pm 0.5 \mathrm{mg} \cdot \mathrm{kgFFM}^{-1} \cdot \mathrm{min}^{-1} ; p<0.05\right)$ and $42 \%$ $\left(8.0 \pm 0.8\right.$ vs $13.6 \pm 1.12 \mathrm{mg} \cdot \mathrm{kgFFM}^{-1} \cdot \mathrm{min}^{-1} ; p<$ 0.002 ) at low and high insulin infusions, respectively.
Similarly, during protocol II, $\mathrm{R}_{\mathrm{d}}$ values remained significantly lower in obese as compared to control children $\left(4.6 \pm 0.4\right.$ vs $6.1 \pm 0.4 \mathrm{mg} \cdot \mathrm{kgFFM}^{-1} \cdot \mathrm{min}^{-1}$; $p<0.05$ at low insulin infusion and $7.4 \pm 0.7 \mathrm{vs}$ $11.3 \pm 0.5 \mathrm{mg} \cdot \mathrm{kgFFM}^{-1} \cdot \mathrm{min}^{-1} ; p<0.002$ at high insulin infusion).

Hepatic glucose release. The dose-response curves of HGR, as reported in Figure 2 (lower panels), suggest that insulin action on HGR is not impaired in obese children. During protocol $I$, no significant differences in the percentage of inhibition of HGR were observed between obese and control children (67 vs $80 \%$ at low insulin infusion; NS; and 97 vs $99 \%$ at high insulin infusion; NS). Interestingly, even if HGR was slightly higher in obese children at low insulin infusion, the differences never reached a statistical significance as compared to obese and control children at high insulin infusion. During protocol II, similar inhibitions of HGR were achieved in obese and control children at low and high insulin infusions ( 39 vs $41 \%$; NS; and 65 vs $59 \%$; NS, respectively). HGR levels were higher in all groups during protocol II as compared to protocol I reaching a significant difference only in control children at low insulin infusion.

Insulin effect on NEFA levels. Figure 3 shows the NEFA profiles during the two protocols. The data of protocol I suggest that NEFA response to insulin is not substantially altered in obese children even if 


\section{Protocol I}
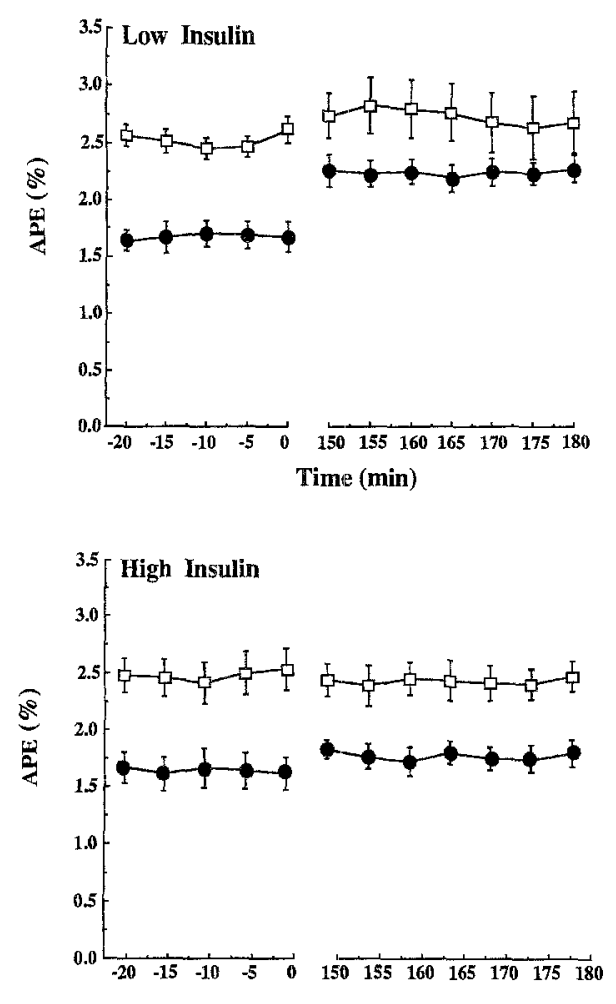

Time (min)

\section{Protocol I}
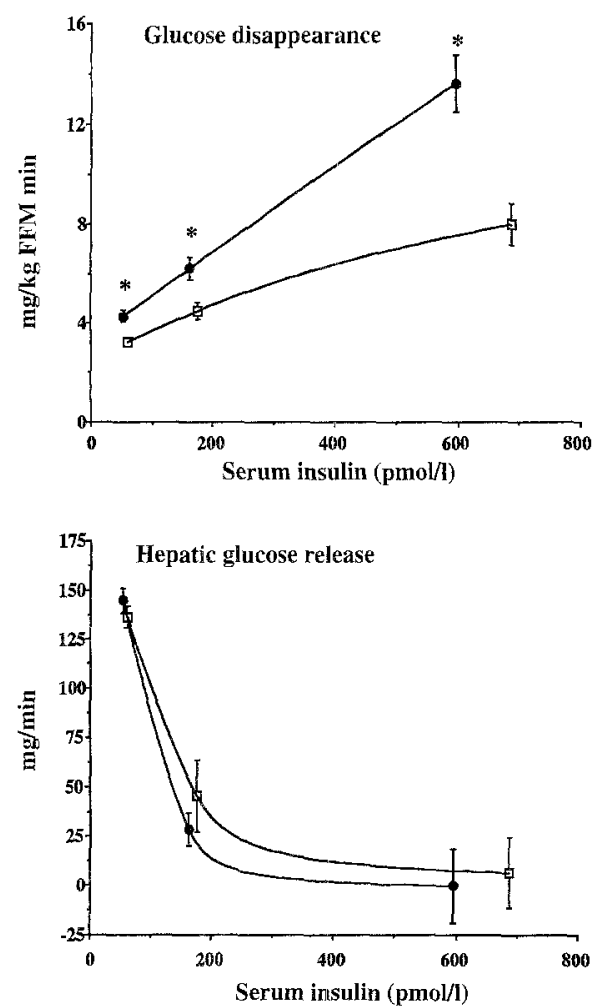

\section{Protocol II}
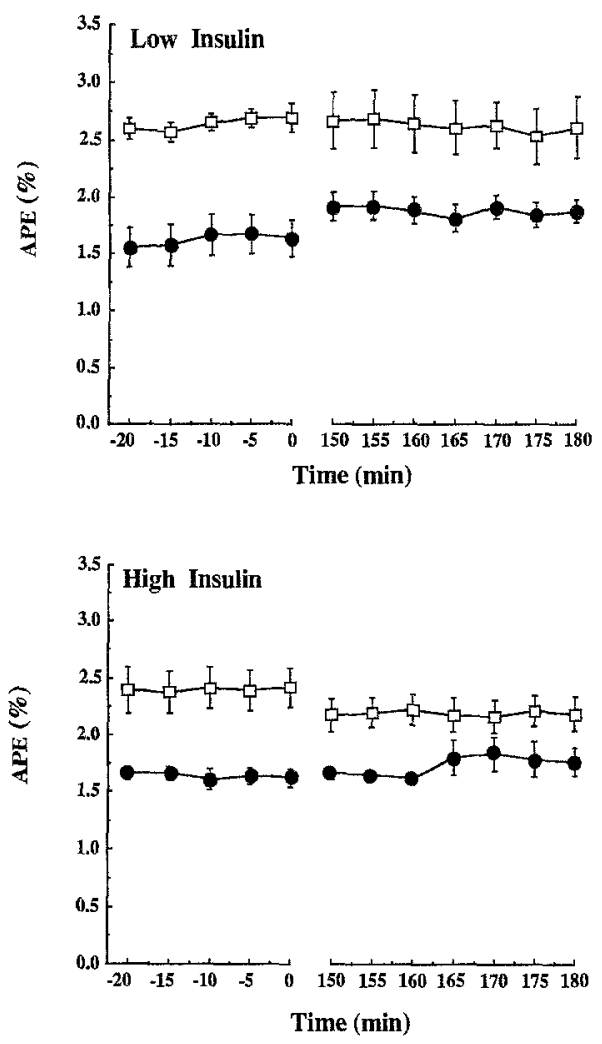

Protocol II
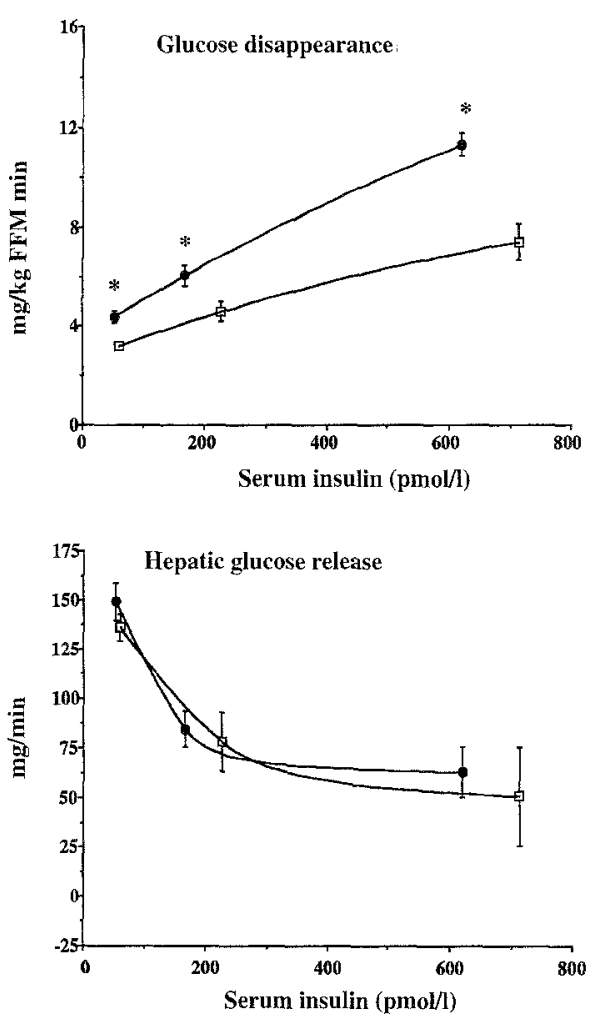

Fig. 1 Time course of changes of APE in the fasting state (from -20 to $0 \mathrm{~min}$.) and during the final portion of the clamp period (from 150 to $180 \mathrm{~min}$ in obese $\square$ and control $\bullet$ children

Fig. 2 Insulin dose-response curves of glucose disappearance and hepatic glucose release during protocols I and II * $p<0.05$ obese $\square$ vs control $\bullet$ children 


\section{Protocol I}
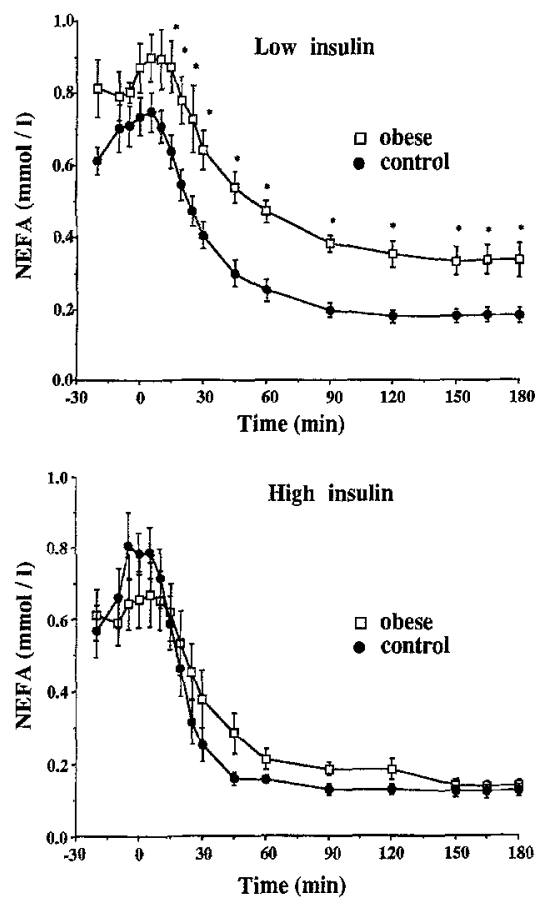

Protocol II
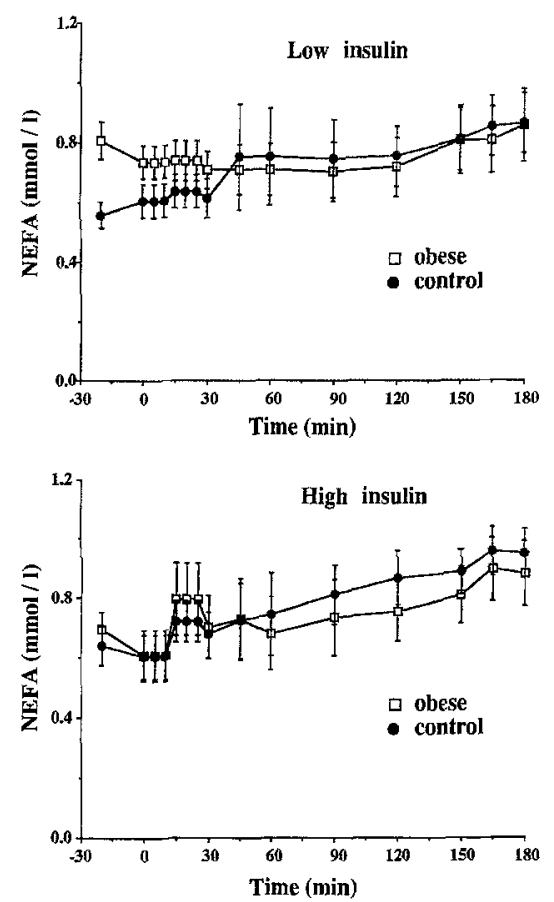

Fig. 3 Time course of NEFA levels during protocols I and II ${ }^{*} p<0.05$ obese $\square$ vs control $\bullet$ children some alterations are already present as reported in Table 3. In obese children both at low or high insulin infusions the time-constant of NEFA decay, $(\mathrm{k})$ was reduced by $30-35 \%$ as compared to values found in control children $(0.034 \pm 0.005$ vs $0.049 \pm 0.009$ and $0.075 \pm 0.019$ vs $0.117 \pm 0.016$ litres $/ \mathrm{min}$; NS). In addition, obese children exhibited a lower degree of inhibition of NEFA levels (plateau levels $=0.33 \pm 0.04$ vs $0.18 \pm 0.02 \mathrm{mmol} / \mathrm{l} ; \quad p<0.05$ and percentages of inhibition $=60.4 \pm 5.3$ vs $75.8 \pm 2.2 \% ; p<0.05$ ) These defects were partially overcome at high insulin levels during which plateau levels were similar and percentages of inhibition were slightly but not significantly lower in obese as compared to control children $(75.8 \pm 4.1$ vs $82.9 \pm 1.8 \%)$. However, the absolute decrements in NEFA were similar between obese and control children both at low $(0.537 \pm 0.082$ vs $0.555 \pm 0.057 \mathrm{mmol} / \mathrm{l} ; \mathrm{NS}$ ) or high insulin infusion $(0.517 \pm 0.086$ vs $0.662 \pm 0.056 \mathrm{mmol} / \mathrm{l} ; \mathrm{NS})$. Glycerol and $\beta$-OH-butyrate levels were similar in the obese and control children and values were superimposable at low or high insulin infusion.

During protocol II, the NEFA levels were maintained constant by a variable lipid infusion. Values were $0.859 \pm 0.12$ and $0.867 \pm 0.1 \mathrm{mmol} / \mathrm{l}$ in obese and control children at low insulin infusion, and $0.883 \pm 0.109$ and $0.952 \pm 0.082 \mathrm{mmol} / 1$ at high insulin infusion. The lipid infusions needed to clamp the NEFA levels in obese children was about $30 \%$ lower those of control children during both insulin infusions $(1.06 \pm 0.008$ vs $1.38 \pm 0.007 \mathrm{ml} / \mathrm{min}, p<0.05$ at low insulin infusion; $2.38 \pm 0.008$ vs $3.54 \pm 0.02 \mathrm{ml} /$ min, $p<0.05$ at high insulin infusion). Glycerol and
$\beta$-OH-butyrate levels significantly increased as compared to the fasting values. The area under the curve of glycerol levels was slightly but not significantly lower in obese than control children while the area under the curve of $\beta$-OH-butyrate was comparable both during low and high insulin infusion.

\section{Discussion}

A point still in debate is whether in simple obesity, hyperinsulinaemia precedes or is preceded by alterations in glucose and lipid metabolism that are usually found in obese subjects. Previous studies $[7,8,21,42$, 43] failed to give a definite answer since the obese subjects studied were already hyperinsulinaemic in the fasting state. To clarify this issue, we studied glucose turnover and lipid levels in a group of obese children with obesity of recent onset but still in the presence of normal fasting insulin levels. Two experimental protocols were performed in the obese children and in a control group of normal-weight children. Protocol I consisted of a euglycaemic, hyperinsulinaemic clamp at two different insulin levels within the physiological range. Protocol II was similar to the first one except for the use of a variable lipid infusion which allowed us to clamp NEFA at the basal levels.

Our data show that, despite normal fasting insulin levels, peripheral insulin action was impaired in obese children. In the basal steady state, a significant reduction of peripheral glucose utilization was present in obese children. In addition, the insulin dose- 
response curve of $R_{d}$ derived from protocol I (Fig. 2, left upper panel) demostrated that obese children suffer from a marked degree of peripheral insulin resistance, reflecting primarily muscle insensitivity to insulin action. These data are consistent with previous studies on obese adults. Bonadonna et al. [7], using the euglycaemic insulin clamp technique within the physiological range of insulin concentrations, found an impairment in insulin-mediated glucose disposal. A marked reduction of glucose disposal was demonstrated in massively obese subjects by Del Prato et al. [8] both in the fasting state and during a euglycaemic clamp.

In contrast to peripheral insulin action, liver sensitivity to insulin was not altered in our obese children. Basal HGR (expressed as the total glucose output) was similar in obese and normal subjects. Furthermore, insulin dose-response curves for suppression of HGR did not differ significantly between the two groups (Fig.2, left lower panel). At insulin levels of $150 \mathrm{pmol} / \mathrm{l}$ a similar decrease of hepatic glucose production was observed in obese and control children achieving values not significantly different from those obtained in the two groups at $600 \mathrm{pmol} / \mathrm{l}$. Interestingly, the values obtained in control children are in striking agreement with those obtained by Katz et al. $[44]$ in normal adults, confirming that the human liver, even during childhood, is exquisitely sensitive to insulin. Our finding that hepatic response to insulin action is not appreciably altered in children at an early stage of obesity is consistent with the results of previous studies on rats at an early stage of obesity [45] or in rats with ventromedial hypothalamic lesions [46]. Furthermore, our finding of a normal liver sensitivity in normoinsulinaemic obese children coupled with the observation of an increased HGR in hyperinsulinaemic obese children, as demonstrated by Bougneres et al. [21], suggests that a defect in the inhibition of hepatic glucose production by insulin develops later in obesity.

The dose-response curves for stimulation of glucose utilization and inhibition of HGR obtained from protocol II provide insight into the effect of NEFA levels on glucose turnover in the obese and control groups. When NEFA levels were clamped at the fasting levels of the obese children in both groups, a significant $25 \%$ reduction in peripheral insulin sensitivity (measured by the ratio between the increments in glucose clearance and insulin level) was demonstrated in control children while no changes were observed in obese children. Our data are similar to previously reported data in control $[16$, $18,19,47]$ and obese adults [17]. As in the case of data from Bevilacqua et al. [17], the lack of decrease of peripheral insulin sensitivity in obese children might be related to an already depressed glucose disposal. In fact, it has been suggested that during an acute intralipid infusion, the degree of inhibition of glucose utilization is related to the overall rate of glucose disposal $[48,49]$. Confirming previous reports [50], lipid infusion determined an increase of HGR, present in all groups, but reaching a statistical significance only in control children at low insulin infusion.

Although fasting NEFA levels were not different between obese and control children, $\beta$-OH-butyrate levels were higher in obese than control children. This might reflect an early resistance of insulin action on hepatic ketogenesis in obese children in keeping with a previously reported direct insulin inhibition of hepatic ketogenesis independent of NEFA supply [51], and in agreement with early metabolic alterations in obese children [52].

The time courses of NEFA levels during protocol I suggest that some alterations in the effect of insulin on NEFA turnover, previously reported in obese adults [53], were present in our obese children. In fact, during protocol I both at low or high insulin the rate of NEFA decay $(\mathrm{k})$ tended to be lower in obese children than in the control group. In addition, the percent decrease in NEFA levels during the low insulin infusion rate (corresponding to an increment of $120 \mathrm{pmol} / \mathrm{l}$ in peripheral insulin) was lower in the obese children (Table 3). Additional evidence of a decreased insulin action on NEFA turnover in obese children is provided by the fact that during protocol II the lipid infusion needed to clamp NEFA levels in obese children was $30 \%$ of that used in control children at both insulin infusion rates, in keeping with a previous study by Bevilacqua et al. [17] in which similar intralipid infusions produced twofold higher NEFA levels in obese as compared to normal adult subjects. Nevertheless, the fact that a high insulin infusion rate resulted in no differences in the percentage of NEFA inhibition and that the absolute decrements in NEFA levels were similar in the two groups at either insulin infusion suggest that defects in insulin action on NEFA levels in the obese group are modest and in an initial stage.

The intimate mechanism(s) of the reduced insulin action on NEFA levels in obese children cannot be ascertained from our data because NEFA production and utilization fluxes were not directly assessed. Thus, further investigation is needed to clarify whether at this early stage of obesity NEFA oxidation is increased or not. Nevertheless, since in obese children NEFA levels during the clamp remained higher than in control children and it has been extensively demonstrated that NEFA oxidation correlates with plasma NEFA concentration [54-56], we speculate that NEFA oxidation may be increased in obese children, which might contribute to insulin resistance. This notion is supported by the presence of an inverse relationship between NEFA levels and peripheral glucose utilization rates during protocol I $(r=0.533 ; p<0.004)$. On the other hand, since the 
acute intralipid infusion used to clamp basal NEFA levels during protocol II did not cause a further decrease in the peripheral insulin sensitivity of the obese group, it is likely that NEFA are only one of the determinants of insulin resistance in obesity. This is also sustained by the fact that in control children an acute increase in FFA levels during insulin stimulation was not sufficient to reproduce the same degree of insulin resistance as in the obese children. Thus, it is likely that some other factors, probably a combination of receptor and post-receptor defects, are present in the induction of insulin resistance of the obese group, as previously suggested $[6,57]$.

In conclusion, the current study provides a portrait of glucose metabolism and lipid levels in obese children with fasting normoinsulinaemia. Our results show that peripheral insulin resistance is the first alteration at this stage of obesity, whereas an increase in insulin secretion and a defect in the inhibition of HGR by insulin may develop at a later stage. Furthermore, primarily receptor and post-receptor defects and some alterations of NEFA metabolism are likely to coexist in the induction of insulin resistance at this stage of obesity.

Acknowledgements. We are indebted to Ms. P. Sandoli, Ms. S. Costa, Ms. P. Van Chuong and Ms. L. Marazzini for their expert technical assistance. We thank Dr. G. Toffolo for her useful suggestions concerning the analysis of stable isotope data.

\section{References}

1. Horton ES, Runge CF, Sims EAM (1970) Endocrine and metabolic effects of experimental obesity in man. Recent Prog Horm Res 29: 457-496

2. Reaven GM, Morre J, Greefield M (1983) Quantification of insulin secretion and in vivo insulin action in non-obese and moderately obese individuals with normal glucose tolerance. Diabetes 32: 600-604

3. Harrison LC, Martin FIR, Melick RA (1976) Correlation between insulin recpetor binding in isolated fat cells and insulin sensitivity in obese human subjects. J Clin Invest 58: 1435-1441

4. Kashiwagi AC, Bogardus C, Lillioja S et al. (1984) In vitro insensitivity of glucose transport and antilipolysis to insulin due to receptor and post-receptor abnormalities in obese Pima Indians with normal glucose tolerance. Metabolism 33: 772-777

5. Kolterman OG, Reaven GM, Olefsky JO (1979) Relationship between in vivo insulin resistance and decreased insulin receptors in obese man. J Clin Endocrinol Metab 48: $487-494$

6. Kolterman OG, Insel J, Saekow M, Olefsky JM (1980) Mechanisms of insulin resistance in human obesity: evidence for receptor and postreceptor defects. J Clin Invest 65: 1272-1284

7. Bonadonna RC, Groop L, Kraemer N et al. (1990) Obesity and insulin resistance in humans: a dose-response study. Metabolism 39: 452-459

8. Del Prato S, Enzi G, Vigili de Kreutzenberg S et al. (1990) Insulin regulation of glucose and lipid metabolism in massive obesity. Diabetologia 33: 228-236
9. Zuniga-Guiardo S, Jimenez J, Angel A, Zinman B (1986) Effects of massive obesity on insulin sensitivity and insulin clearance and the metabolic response to insulin as assessed by the euglycaemic clamp technique. Metabolism 35: $278-282$

10. Randle PJ, Garland PB, Hales CN, Newsholme EA (1963) The glucose-fatty acid cycle. Its role in insulin sensitivity and the metabolic disturbances of diabetes mellitus. Lancet 1: 785-789

11. Randle PJ, Newsholme EA, Garland PB (1964) Regulation of glucose uptake by muscle. Effects of fatty acids, ketone bodies and pyruvate, and alloxan-diabetes and starvation, on the uptake and metabolic fate of glucose in rat heart and diaphragm muscle. Biochem J 93: 652-687

12. Lillioja S, Foley J, Bogardus C et al. (1986) Free fatty acid metabolism and obesity in man: in vivo and in vitro comparisons. Metabolism 35: 505-514

13. Piatti PM, Monti LD, Pacchioni M, et al. (1991) Forearm insulin- and non-insulin-mediated glucose uptake and muscle metabolism in man: role of free fatty acids and blood glucose levels. Metabolism 40: 926-933

14. Vouillamoz D, Temler E, Jequier E, Felber JP (1987) Importance of substrate competition in the mechanism of insulin resistance in man. Metabolism 36: 715-720

15. Ferrannini E, Barrett EG, Bevilacqua S, DeFronzo RA (1983) Effects of fatty acids on glucose production and utilization in man. J Clin Invest 72: 1737-1747

16. Balasse EA, Neef MA (1974) Operation of the glucose-fatty acid cycle during experimental elevations of plasma free fatty acid levels in man. Eur J Clin Invest 4: 247-252

17. Bevilacqua S, Bonadonna R, Buzzigoli $G$ et al. (1987) Acute elevation of free fatty acid levels leads to hepatic resistance in obese subjects. Metabolism 36: 502-506

18. Thiebaud D, De Fronzo RA, Jacot E et al. (1982) Effect of long chain triglyceride infusion on glucose metabolism in man. Metab Clin Exp 31: 1128-1136

19. Felber JP, Ferrannini E, Golay A et al. (1987) Role of lipid oxidation in pathogenesis of insulin resistance of obesity and type II diabetes. Diabetes 36: 1341-1350

20. Golay A, Felber JP, Meyer HU et al. (1984) Study on lipid metabolism in obesity and diabetes. Metabolism 33:111-116

21. Bougneres PF, Artavia-Loria E, Henry S et al. (1989) Increased basal glucose production and utilization in children with recent obesity versus aduit with long-term obesity. Diabetes 38: 477-483

22. Le Stunff C, Bougneres PF (1993) Time course of increased lipid and decreased glucose oxidation during early phase of childhood obesity. Diabetes 42: 1010-1016

23. Amiel SA, Sherwin RS, Simonson DC et al. (1986) Impaired insulin action in puberty: a contributing factor to poor glycemic control in adolescents with diabetes. N Engl J Med 315: 215-219

24. Bloch CA, Clemons P, Sperling MA (1987) Puberty decreases insulin sensitivity. J Pediatr 110: 481-487

25. Tanner J, Whitehouse RH (1976) Clinical longitudinal standards for height, weight and weight velocity and stages of puberty. Arch Dis Child 51: 170

26. Hernesniemi I, Zachmann M, Prader A (1974) Skinfold thickness in infancy and adolescence: a longitudinal correlation study in normal children. Helv Paediatr Acta 29:523-528

27. Wormersley I, Durning JVGA (1977) A comparison of the skinfold method with extent of "overweight" and various weight-height relationships in the assessment of obesity. Br J Nutr 38: 271-284

28. Brook CGD (1971) Determination of body composition of children from skinfold measurements. Arch Dis Child 46: 182-184 
29. Istituto Nazionale della Nutrizione (1989) Livelli di assunzione giornalieri raccomandati di energia e di nutrimenti per la popolazione italiana. Revisione 1986-87 e tabella di composizione degli alimenti. Milano: Litho Delta Ed.

30. Beer SF, Heaton DA, Alberti KGMM, Pyke DA, Leslie RDG (1990) Impaired glucose tolerance precedes but does not predict insulin-dependent diabetes mellitus: a study of identical twins. Diabetologia 33: 497-502

31. Pettitt DJ, Moll PP, Knowler WC et al. (1993) Insulinemia in children at low and high risk of NIDDM. Diabetes Care 16: 608-615

32. National Diabetes Data Group (1979) Classification and diagnosis of diabetes mellitus and other categories of glucose intolerance. Diabetes 28: 1039-1057

33. Finegood DT, Bergman RN, Vranic M (1987) Estimation of endogenous glucose production during hyperinsulinemic-euglycaemic glucose clamps: comparison of unabeled and labeled exogenous glucose infusates. Diabetes 36: 914-924

34. Steel R (1959) Influences of glucose loading and injected insulin on hepatic glucose output. Ann NY Acad Sci 82: $420-430$

35. Cobelli C, Toffolo G, Foster DM (1992) Tracer-to-tracee ratio for the analysis of stable isotope tracer data:the link with the radioactive kinetic formalism. Am J Physiol 262: E968-E975

36. Finegood DT, Bergman RN (1983) Optimal segments: a method for smoothing tracer data to calculate metabolic fluxes. Am J Physiol 244: E471-E479

37. Coon PJ, Rogus EM, Goldberg AP (1992) Time course of plasma free fatty acid concentration in response to insulin: effect of obesity and physical fitness. Metabolism 41: 711716

38. Carson ER, Cobelli C, Finkelstein L (1983) The mathematical modeling of metabolic and endocrine systems. Wiley, New York

39. Magni F, Monti LD, Brambilla P et al. (1992) Determination of plasma $(6,62 \mathrm{H} 2)$-glucose enrichment by a simple and accurate gas chromatographyc mass spectrometric method. J Chromatography 573: 127-131

40. Knox DP, Jones DG (1984) Automated enzymatic determination of plasma free fatty acids by centrifugal analysis $J$ Autom Chem 6: 152-154

41. Monti LD, Sandoli E, Costa S et al. (1993) Fluorimetric methods for the measurement of intermediate metabolites (lactate, pyruvate, alanine, $\beta$-hydroxybutyrate, glycerol) using a Cobas Fara centrifugal analyser. J Autom Chem 15: $177-181$

42. Gorden ES (1960) Non-esterified fatty acids in the blood of obese and lean subjects. Am J Clin Nutr 8: 740-747
43. Opie LH, Walfish PG (1963) Plasma free fatty acid concentrations in obesity. N Engl J Med 268: 756-760

44. Katz H, Butler P, Homan M et al. (1993) Hepatic and extrahepatic insulin action in humans: measurement in the absence of non-steady-state error. Am J Physiol 264: E561E566

45. Penicaud L, Ferré P, Terretaz J et al. (1987) Early insulin resistance in muscles but normal sensitivity in white adipose tissue. Diabetes 36: 626-631

46. Penicaud L, Rohner-Jeanrenaud F, Jeanrenaud B (1986) In vivo metabolic changes as studied longitudinally after ventromedial hypothalamic lesions. Am J Physiol 250: E662-E668

47. Lee KU, Lee HK, Koh CS, Min HK (1988) Artificial induction of intravascular lipolysis by lipid-heparin infusion leads to insulin resistance in man. Diabetologia 31:285-290

48. Ferrannini E, Barrett EJ, Bevilacqua S et al. (1983) Effect of fatty acids on glucose production and utilization in man. J Clin Invest 72: 1737-1747

49. Bonadonna RC, Zych K, Boni C, Ferrannini E, DeFronzo RA (1989) Time dependence of the interaction between lipid and glucose in humans. Am J Physiol 257: E49-E56

50. Boden G, Chen X, Ruiz J, White JV, Rossetti L (1994) Mechanisms of fatty acid-induced inhibition of glucose uptake. J Clin Invest 93: 2438-2446

51. Keller V, Chiasson JL, Liljenquist JE et al. (1977) The roles of insulin, glucagon, and free fatty acids in the regulation of ketogenesis in dogs. Diabetes 26: 1040-1051

52. Berenson GS, Radhakrishnamurthy B, Srinivasan SR et al. (1981) Plasma glucose and insulin levels in relation to cardiovascular risk factors in children from biracial population - the Bogalusa heart study. J Chron Dis 34: 379-391

53. Groop L C, Saloranta C, Shank M et al. (1991) The role of free fatty acid metabolism in the pathogenesis of insulin resistance in obesity and noninsulin-dependent diabetes mellitus. J Clin Endocrinol Metab 72: 96-107

54. Howard BV, Klimes I, Vasquez B et al. (1984) The antilipolytic action of insulin in obese subjects with resistance to its glucoregulatory action. J Clin Endocrinol Metab 58: $544-548$

55. Campbell PJ, Carlson MG, Nurjhan N (1994) Fat metabolism in human obesity. Am J Physiol 266: E600-E605

56. Bonadonna R, Groop LC, Zych K, Shank M, DeFronzo RA (1990) Dose-dependent effect of insulin on plasma free fatty acid turnover and oxidation in humans. Am J Physiol 259: E736-E750

57. Olefsky JM (1981) Insulin resistance and insulin action: an in vitro and in vivo perspective. Diabetes 30: 148-162 